LSG zu dem Schluss, dass die geplante häusliche Eigenanwendung des Medizinprodukts nicht geeignet und erforderlich sei, den Erfolg der Krankenbehandlung zu sichern, so dass das LSG einen Anordnungsanspruch verneinte und die Beschwerde des Versicherten gegen den erstinstanzlichen Beschluss zurückwies.

\section{b) Hilfsmitteleinsatz im Rahmen einer neuen Untersuchungs- und Behandlungsmethode}

Der Gemeinsame Bundesausschuss (G-BA) beschloss 2017 eine Richtlinie ${ }^{19}$ zur Erprobung der Transkorneale Elektrostimulation zur Behandlung der Retinitis Pigmentosa (erblich bedingte Erkrankung der Netzhaut). Obwohl das Erprobungsverfahren noch nicht abgeschlossen war und somit die Anerkennung dieser neuen Methode durch den GBA fehlte, bejahte das LSG Baden-Württemberg, wie in der letzten Rechtsprechungsübersicht erwähnt ${ }^{20}$, den Anspruch der klagenden Versicherten auf eine Versorgung mit dem bei der Behandlungsmethode zum Einsatz kommenden OkuStim ${ }^{\circledR}$ System (bestehend aus Gerät, Brille und Elektroden). Das LSG sah im Fall einer klagenden Versicherten einen Ausnahmefall gem. \$2 Abs. 1a SGB V als gegeben an. Die Beschwerde der Beklagten gegen die Nichtzulassung der Revision verwarf das BSG mangels Darlegung einer Rechtsprechungsdivergenz als unzulässig ${ }^{21}$.

\section{c) Hilfsmittelverzeichnis $\int 139$ SGB V}

Die letzte Rechtsprechungsübersicht zum Medizinprodukterecht beinhaltete den Hinweis auf die Entscheidung des LSG Berlin-Brandenburg, nach der die klagende Herstellerin keinen Anspruch auf Aufnahme ihrer Spezialhausschuhe für Diabetiker in das Hilfsmittelverzeichnis hat, weil die Herstellerin den Nachweis der Funktionstauglichkeit und
Sicherheit der streitigen Hausschuhe nicht erbracht hatte ${ }^{22}$. Die Nichtzulassungsbeschwerde der Klägerin verwarf das BSG wegen nicht ausreichender Darlegung eines Zulassungsgrundes als unzulässig ${ }^{23}$.

In einem einstweiligen Verfahren vor dem SG München ${ }^{24}$ und Bay. $\mathrm{LSG}^{25}$ ging es um das im Hilfsmittelverzeichnis gelistete PleurX Peritoneal-Kathetersystem, das außerhalb des vorgesehenen Indikationsbereichs zum Einsatz kommen sollte. Lt. Hilfsmittelverzeichnis ist das Kathetersystem für die Pleuraerguss-Drainage bei moribunden Patienten mit Pleuracarcinose indiziert. Beim Antragsteller sollte es zur Behandlung einer pulmonalen Hypertonie mit stauungsbedingter Leberzirrhose und Aszites eingesetzt werden. Das SG führte aus, dass es sich beim Einsatz eines Produkts in einem nicht im Hilfsmittelverzeichnis enthaltenen Indikationsbereich, um einen Einsatz im Rahmen einer neuen Behandlungsmethode handele. Für diese neue Methode fehle die Anerkennung durch den G-BA, so dass der Antragsteller keinen Anspruch auf das Kathetersystem habe. Die gegen den Beschluss des SG gerichtete Beschwerde des Antragstellers wies das LSG zurück und begründete seine Entscheidung zudem mit vorhandenen Behandlungsalternativen.

19) Erprobungs-Richtlinie TES-RP vom 20.7.2017, BAnz AT 6.10.2017 B3, https://www.g-ba.de/richtlinien/99/.

20) LSG Bad.-Württ., Urt. v. 18.2.2020 - L 11 KR 2478/19 -, juris; Vorjahresbericht Hobusch/Ochs, MedR 2020, 911 ff., sub 5. b).

21) BSG, Beschl. v. 27.10.2020 - B $3 \mathrm{KR} 18 / 20 \mathrm{~B}-$, juris.

22) Vorjahresbericht Hobusch/Ochs, MedR 2020, 911 ff., sub 5. c).

23) BSG, Urt. v. 13.1.2021 - B 3 KR 10/20 B -, juris.

24) SG München, Beschl. v. 19.3.2020 - S 19 KR 279/20 ER BeckRS 2020, 12629.

25) Bay. LSG, Beschl. v. 10.6.2020 - L 4 KR 150/20 B ER BeckRS 2020, 12627.

\title{
Der Aufbau der Europäischen Gesundheitsunion - Lernen aus der Corona-Krise
}

\section{Karin Henke}

\begin{abstract}
:
Der Beitrag setzt an der Tagung des ineges v. 22.3.21 zum Thema „Ein Jahr Corona: Welche Lehren zieht das Mehrebenensystem? Zur Krisenresilienz und Alltagstanglichkeit des Gesundheitswesens." an. Die Verfasserin beschäftigt sich mit der Frage, welche Lehren aus der Corona-Krise gezogen wurden und inwiefern diese in den Aufbau der Europäischen Gesundheitsunion eingeflossen sind. Dabei werden die Handlungs- und Koordinierungsprobleme des Gesundheitssystems auf europäischer Ebene aufgezeigt und den neuen Legislativprojekten gegenübergestellt.
\end{abstract}

Karin Henke, wissenschaftliche Mitarbeiterin am ineges Institut für Europäische Gesundheitspolitik und Sozialrecht, promoviert zum Thema „Möglichkeiten und Grenzen für Erstattungsansprüche von Liposuktionen bei Lipödem“ bei Doktormutter: Prof. Dr. iur Astrid Wallrabenstein, Richterin des BVerfG, Gebäude RuW (Recht und Wirtschaft), Raum 3.126, Theodor-W.-Adorno-Platz 4, 60629 Frankfurt am Main, Deutschland
Die deutsche Gesundheitspolitik fußt auf dem politisch geprägten Gesundheitswesen und versteht sich als politische Querschnittsaufgabe ${ }^{1}$. Nach Busch hat sich Gesundheitspolitik lediglich auf Bereiche des körperlichen und psychischen Wohlbefindens zu beschränken ${ }^{2}$, soziale Rahmenbedingungen bleiben dabei außen vor ${ }^{3}$. Die Gesundheitspolitik wurde stets als nationale Aufgabe gesehen, jegliches Handeln fußte auf dieser Sichtweise. Das Auftreten des COVID-19-Virus und dessen Verbreitung über Ländergrenzen und Kontinente hinweg erfordert eine neue Denk-

1) König, Gesundheit, Gesundheitspolitik und soziale Gerechtigkeit: Überlegungen zu einer gesundheits-zentrierten Gesundheitspolitik, Hamburg, 2011, S. $131 \mathrm{ff}$.; Rosenbrock/Gerlinger, Gesundheitspolitik, 3. Aufl. 2014, S. 15 f.

2) Berg, Gesundheitsschutz als Aufgabe der EU: Entwicklung, Kompetenzen, Perspektiven, 1997, S. $60 \mathrm{ff}$; Schmidt am Busch, Die Gesundheitssicherung im Mehrebenensystem, 2007, S. 9.

3) Wallrabenstein, in: Schlachter/Heinig, Europäisches Arbeits- und Sozialrecht, Enzyklopädie Europarecht, Band 8, 2. Aufl. 2020, §8 Gesundheitspolitik, Rdnr. 2. 
weise und daraus resultierend ein über die bisherige Praxis hinausgehendes Handeln. Grenzüberschreitendem Geschehen muss grenzüberschreitend begegnet werden. Dies ist nicht aus dem Stand heraus erfolgreich zu bewältigen, es bedarf der gründlichen Analyse der bisherigen Ereignisse und eines länderübergreifenden Handlungskonzepts ${ }^{4}$. Ausgehend davon plant die Europäische Kommission eine Europäische Gesundheitsunion. Schwerpunkt der Arbeit sind die Weiterentwicklung der Kompetenzen in der Gesundheitspolitik und der Ausbau der Koordinierung zwischen den Mitgliedsstaaten ${ }^{5}$. Grundlage dafür ist die Analyse der auf europäischer Ebene sichtbar gewordenen Handlungsund Koordinierungsprobleme ${ }^{6}$. Die darauf basierenden Vorschläge bilden die Eckpfeiler der Europäischen Gesundheitsunion einschließlich der Legislativprojekte, welche aus der Corona-Krise resultieren.

\section{A. Bisherige Kontrollinstrumente zur Wahrung der Gesundheitspolitik}

Die Gesundheitspolitik in Deutschland wurde bis zur Covid-19-Pandemie primär von nationalen Themen geleitet und war demzufolge nicht auf die Harmonisierung der Rechtsvorschriften der Mitgliedstaaten gerichtet. Ungeachtet dessen gab es einige Kooperationen. Beispiel dafür ist der im Jahr 2013 erlassene Beschluss zu schwerwiegenden grenzüberschreitenden Gesundheitsgefahren ${ }^{7}$. Er bildet bereits einen Rechtsrahmen zum Umgang mit pandemieähnlichen Situationen auf europäischer Ebene ${ }^{8}$. Kernelement ist das Ergreifen gemeinsamer medizinischer Gegenmaßnahmen bei grenzüberschreitenden Gesundheitsbedrohungen ${ }^{9}$. Der Schwerpunkt liegt dabei auf der grenzüberschreitenden Zusammenarbeit und Koordinierung der Mitgliedsstaaten zur besseren Prävention und Kontrolle hinsichtlich schwerer, den Menschen betreffenden, Krankheiten ${ }^{10}$.

Dabei sollte die Zusammenarbeit und Koordinierung zwischen den Mitgliedstaaten im Interesse einer besseren Prävention und Kontrolle der Ausbreitung schwerer Krankheiten über die Grenzen der Mitgliedstaaten hinaus, unterstützt werden. Ein weiteres Ziel der Zusammenarbeit ist die Bekämpfung anderer schwerwiegender grenzüberschreitender Gesundheitsgefahren sowie die Aufrechterhaltung eines hohen Gesundheitsschutzniveaus in der Union ${ }^{11}$. Dabei ist der diesbezügliche Handlungsspielraum jedoch beschränkt. Der derzeitige Beschluss enthält jedoch bereits erste rechtliche Regelungen zur gemeinsamen Beschaffung medizinischer Gegenmaßnahmen $^{12}$, zur ad-hoc Beobachtung ${ }^{13}$ und zur Einrichtung eines Frühwarn- und Reaktionssystems ${ }^{14}$. Hinzu kommt die Tätigkeit der bereits bestehenden EU-Agenturen. Diese dienen der koordinierten Reaktion auf grenzüberschreitende Gesundheitsgefahren und tragen zur Wahrung der Gesundheitssicherheit innerhalb der EU bei ${ }^{15}$. EU-Agenturen sind das Europäische Zentrum für Prävention und Kontrolle von Krankheiten (ECDC) und die Europäische Arzneimittel-Agentur (EMA) ${ }^{16}$. Die Aufgabe des ECDC besteht in der Verbesserung der Verhütung und Kontrolle übertragbarer Krankheiten in der Europäischen Union. Es prüft gesundheitliche Bedrohungslagen über die nationalen Grenzen hinweg und informiert die Mitgliedsstaaten wechselseitig ${ }^{17}$. Die EMA prüft und bewertet Human- und Tierarzneimittel; sie entscheidet über deren EU-weite Zulassung ${ }^{18}$.

Die Pandemiesituation hat aufgezeigt, dass die bestehenden Strukturen krisenanfällig sind. Deshalb werden Maßnahmen ergriffen, um zukünftige Pandemien verbessert bewältigen zu können.

\section{B. Auswirkungen der Corona-Krise auf die Zusammenarbeit im Gesundheitswesen}

Die Union und die Mitgliedsstaaten fördern die Zusammenarbeit mit den für das Gesundheitswesen zuständigen internationalen Behörden, um ein hohes Gesundheitsschutzniveau sicherzustellen ${ }^{19}$. Ein Ziel ist unter anderem der Schutz und die Verbesserung der menschlichen Gesundheit $^{20}$, wobei der Focus auf dem Bereich der Public Health im Sinne einer allgemeinen öffentlichen Gesundheit liegt ${ }^{21}$. Ungeachtet der zahlreich vorhandenen Sicherheitsinstrumente verlief die auf EU-Ebene angestrebte gemeinsame Reaktion auf den Ernstfall (Pandemie) nicht erwartungsgemäß. Die Effizienz der Sicherheitsinstrumente ist hinsichtlich Geschwindigkeit und Passfähigkeit länderübergreifend zu optimieren, wobei landes-egoistische Tendenzen wie Ausfuhrbeschränkungen und Einbehaltung wichtiger medizinischer Ressourcen der Vergangenheit angehören sollten. Gleiches gilt für unabgestimmte Grenzkontrollen sowie Schließungen von EU-Binnengrenzen ${ }^{22}$.

Es ist den einzelnen Ländern überlassen, welche Maßnahmen bei Eintreten des Ernstfalles ergriffen werden, wobei ein EU-weiter Austausch diesbezüglich anzustreben ist. Als Beispiel mag der länderübergreifende Transport der zu beatmenden COVID-19-Patienten dienen.

$\mathrm{Zu}$ Beginn der Pandemie war das Handeln der Mitgliedsstaaten geprägt von Ausfuhrbeschränkungen, Einbehaltung wichtiger medizinischer Ressourcen und Kontrollen der Binnengrenzen. In Deutschland wurde dem Pandemie-Geschehen mit zahlreichen Maßnahmen begegnet. Genannt seien hierfür Kontaktbeschränkungen, Ausgangsperren, HomeOffice-Präferenz, Schul- und Kita-Schließungen sowie Maßnahmen zur Reduzierung oder Unterbindung von Gruppenkontakten ${ }^{23}$. Staatlicherseits wurden Gelder eingesetzt, um einer Insolvenz von Betrieben vorzubeu-

4) Europäische Kommission, Schaffung einer europäischen Gesundheitsunion: Stärkung der Krisenvorsorge und -reaktion für Europa, Pressemitteilung v. 11.11.2020 (EUCom, Pressemitteilung v. 11.11.2020).

5) Mussler, EU will in Gesundheitspolitik enger zusammenarbeiten“, FAZ, aktualisiert am 11.11.2020, https://www.faz.net/aktuell/politik/ausland/eu-will-in-gesundheitspolitik-enger-zusammenarbeiten-17047702.html, letzter Zugriff am 16.6.2021.

6) Mussler, EU will in Gesundheitspolitik enger zusammenarbeiten", FAZ, aktualisiert am 11.11.2020, https://www.faz. net/aktuell/politik/ausland/eu-will-in-gesundheitspolitikenger-zusammenarbeiten-17047702.html, letzter Zugriff am 16.6. 2021.

7) Beschl. (EU) Nr. 1082/2013 des Europäischen Parlaments und des Rates v. 22.10.2013 zu schwerwiegenden grenzüberschreitenden Gesundheitsgefahren, ABl. Nr. L 293/1. v. 5. 11.2013.

8) EUCom, Pressemitteilung v. 11.11.2020.

9) Vorerklärung (1), Beschl. Nr. 1082/2013/EU.

10) Art. 1 Abs. 2, Beschl. Nr. 1082/2013/EU.

11) Art. 1 Abs. 2, Beschl. Nr. 1082/2013/EU.

12) Art. 5 Abs. 1, Beschl. Nr. 1082/2013/EU.

13) Art. 7 Abs. 1, Beschl. Nr. 1082/2013/EU.

14) Art. 8 Abs. 1, Beschl. Nr. 1082/2013/EU.

15) Mitteilung der Kommission an das Europäische Parlament, den Rat, den Europäischen Wirtschafts- und Sozialausschuss und den Ausschuss der Regionen, COM(2020) 724 final, S. 6.

16) EUCom, Pressemitteilung v. 11.11.2020.

17) Europäisches Zentrum für Prävention und die Kontrolle von Krankheiten, Die Gesundheit in Europa erhalten, ECDC in Aktion, DOI 10.2900/11971, S. $14 \mathrm{f}$.

18) European Medicines Agency, Das Europäische Arzneimittelregulierungssystem, Ein einheitlicher Ansatz für die Zulassung von Arzneimitteln in der Europäischen Union, EMA/716925/2016, S. 2.

19) Art. 168 AEUV.

20) Büttner/Lemor, in: Sodan, Handbuch des Krankenversicherungsrechts, \$3 Europarechtliche Grundlagen der Krankenversicherung, Rdnr. 10, 3. Aufl. 2018.

21) von Bogdandy/Villarreal, ZaöRV 2020, 293, 300.

22) $\operatorname{COM}(2020) 724$ final, S. 2.

23) Kämmerer/Jischowski, GesR 2020, 341, 345 ff. zu Social Distancing und Einreiseverboten; s. auch: Groth, jurisPR-SozR 7/2020, Anm. 1, S. 1 
gen $^{24}$. Das deutsche Gesundheitswesen war von der durch Covid-19 entstandenen Situation in besonderem Maße betroffen. Teilweise nahm die Kurzarbeit ${ }^{25} \mathrm{zu}-$ auch im Gesundheitswesen - weil die einzelnen Gesundheitsbereiche nur noch notfallmäßig von Patienten aufgesucht wurden, da die Angst vor eine Corona-Infektion sehr hoch war ${ }^{26}$. In anderen Bereichen wurde durch den Gesetzgeber gezielt veranlasst alle Behandlungen außer Notfallbehandlungen auszusetzen ${ }^{27}$. Ziel war, trotz einer hohen Belastung durch Corona-Infizierte eine funktionierende Gesundheitsversorgung sicherzustellen und gleichzeitig einer übermäßigen Belastung des medizinischen Personals vorzubeugen ${ }^{28}$. Eine Pandemie wie die von Covid-19 zeigt die Grenzen der Belastbarkeit auf; dies gilt für das Gesundheitssystem als Ganzes als auch für dessen medizinisches Fachpersonal ${ }^{29}$.

So wie das deutsche Gesundheitssystem, stießen auch EU und Europäische Kommission an ihre Belastungsgrenzen, zumal die EU bezüglich des Gesundheitswesens nur schwach ausgeprägte Kompetenzen hat und zumeist nur unterstützend und koordinierend tätig werden darf ${ }^{30}$. Die Bewältigung einer solchen Krise kann jedoch nur gelingen, wenn nachbarschaftliche Zusammenarbeit über Landesgrenzen hinaus gefördert wird und gemeinsamer Ressourcenüberschuss wie Ressourcenmangel ausgeglichen werden $^{31}$. Der Kommission fehlen jedoch die Befugnisse, auf europäischer Ebene umfassende Maßnahmen zur Koordinierung von Aufgaben zum Schutze der Gesundheit durchzusetzen $^{32}$. Hinzu kommt, dass die derzeitige Vorsorge- und Reaktionsplanung erhebliche Schwächen aufweist ${ }^{33}$. Der Informationsaustausch auf europäischer Ebene konnte keine aufeinander abgestimmte Reaktion erwirken, auch wurde die Risikokommunikation nicht umfangreich ausgeübt ${ }^{34}$. Dies hatte zur Folge, dass der Umgang mit dem Geschehen an den meisten Stellen ein lediglich reaktiver war ${ }^{35}$.

Für einen einheitlichen Umgang mit Gesundheitsmaßnahmen gab es zu große Umsetzungs- und Kommunikationsprobleme ${ }^{36}$. Innerhalb der Europäischen Kommission existieren ein EU-Gesundheitssicherheitsausschuss (HSC) und das Frühwarn- und Reaktionssystem der Gemeinschaft $\left(\right.$ EWRS) ${ }^{37}$. In der Corona-Krise war der HSC für die Mitgliedsstaaten die erste Anlaufstelle zur Risiko- und Krisenkommunikation, so dass aktuelle Maßnahmen und Folgeschritte besprochen werden konnten ${ }^{38}$. Letztlich war der HSC in Ermangelung von Kompetenzen in seiner Umsetzungs- und Kontrollfunktion stark eingeschränkt ${ }^{39}$. Hinzu kamen fehlende Abstimmungen bezüglich des Einsatzes von Masken, Abstandsregelungen (social distancing), sowie des Umgangs mit Personen, welche Risikokontakt hatten (Isolierung und Quarantäne) ${ }^{40}$. Außerdem fehlte eine einheitliche Teststrategie. Praktische Umsetzungsprobleme zeigten sich zum einen an den fehlenden Testkapazitäten, zum anderen war die Kontaktnachverfolgung weder schnell noch effizient. Letzteres ist auf fehlende Absprachen und ungenügende personelle Kapazität zurückzuführen ${ }^{41}$. Schutzausrüstung zur Einhaltung von Hygienemaßnahmen und Sicherheitsstandards war nicht in ausreichendem Maße vorhanden ${ }^{42}$, es fehlte an Vorräten für medizinische Gegenmaßnahmen und die Lieferketten waren störanfällig, so dass es zu vielfältigen Engpässen $\mathrm{kam}^{43}$.

Es ist anzunehmen, dass es auch zukünftig sowohl an medizinischer Schutzrüstung und Medikamenten als auch an Rohstoffen, Materialien und Komponenten für eine eigenständige Medikamenten-Herstellung fehlen wird ${ }^{44}$. Im Jahr 2020 führte die stetig wachsende weltweite Nachfrage im Kontext limitierter Herstellungskapazitäten ${ }^{45}$ beim Bundesinstitut für Arzneimittel und Medizinprodukte (BfArM) zu deutlichen Arzneimittel-Lieferengpässen ${ }^{46}$. Zurückzuführen waren diese zum einen auf eine unzureichende Koordinierung und zum anderen auf das Fehlen eines systematischen Vorgehens im Hinblick auf Beschaffung und Ankauf erforderlicher Impfstoffe, Medikamente und persönlicher Schutzausrüstung (PSA) ${ }^{47}$. Die Vermutung liegt nahe, dass es bei Fortbestand der Corona-Situation weiterhin zu Lieferabrissen kommt und der entsprechende Handlungsbedarf bestehen bleibt ${ }^{48}$.

Bereits in der Vergangenheit, d. h. vor Auftreten der Covid-19-Pandemie, trat die Europäische Union für ein hohes Gesundheitsschutzniveau der in der EU vereinigten Bürger $e^{2 i n}{ }^{49}$ und die Mitgliedsstaaten setzten sich gemeinsam den Schutz der Bevölkerung zum Ziel ${ }^{50}$. Die Grundstrukturen sind vorhanden, es bedarf jedoch der Beseitigung bestehender Schwachstellen. Die Bildung einer Europäischen Gesundheitsunion dient diesem Ziel.

Das EU-Recht darf grundsätzlich keine Harmonisierung der Rechtsvorschriften der einzelnen Mitgliedsstaaten bewirken ${ }^{51}$. Die EU-Kompetenz beschränkt sich auf Maßnahmen zur Festlegung hoher Qualitäts- und Sicherheitsstandards für Organe und Substanzen menschlichen Ursprungs sowie für Blut und Blutderivate ${ }^{52}$. Ebenso schließt es Maßnahmen in den Bereichen Veterinärwesen und Pflanzenschutz, die unmittelbar den Schutz der Gesundheit der Bevölkerung zum Ziel haben ${ }^{53}$ und Maßnahmen zur Festlegung hoher Qualitäts- und Sicherheitsstandards für Arzneimittel und Medizinprodukte mit ein ${ }^{54}$. Über die Ausgestaltung des nationalen Gesundheitswesens entscheidet jeder Mitgliedsstaat selbst. Systemunterschiede in der Ausgestaltung der Gesundheitsversorgung sind dabei unvermeidlich ${ }^{55}$. Das betrifft vor allem die klinische

24) Schneider, COVur 2020, 576, 577.

25) Schneider, COVur 2020, 576, 577.

26) Oberhofer, Drohende Versorgungskrise. Aus Angst vor Corona nicht zum Arzt?, v. 23.4.2020, abrufbar unter https://www. springermedizin.de/covid-19/diagnostik-in-der-infektiologie/ aus-angst-vor-corona-nicht-zum-arzt-/17921188, letzter Zugriff am 15.6.2021.

27) Bockholdt/Lungstras/Schmidt, NZS 2020, 324.

28) Die Kosten für die Nichtbelegung der Stationen wurden durch eine sog. Freihaltepauschale abgerechnet, $\$ 21$ Abs. 1-4 KHG; Schneider, COVur 2020, 576, 578.

29) $\operatorname{COM}(2020) 724$ final, S. 1.

30) Lurger, in: Streinz, EUV/AEUV, AEUV, 3. Aufl.2018, Art. 168, Rdnr. 48.

31) $\operatorname{COM}(2020) 724$ final, S. 2.

32) Deutsche Sozialversicherung Europavertretung, Mandat der EMA soll gestärkt werden, 1.12.2020, https://dsv-europa.de/de/ news $/ 2020 / 11 /$ mandat-der-ema-soll-gestaerkt-werden.html, letzter Zugriff am 1.12.2020, S. 1.

33) $\operatorname{COM}(2020) 724$ final, S. 5.

34) $\operatorname{COM}(2020) 724$ final, S. 5.

35) Deutsche Sozialversicherung Europavertretung, Mandat der EMA soll gestärkt werden, 1.12.2020, https://dsv-europa.de/de/ news/2020/11/mandat-der-ema-soll-gestaerkt-werden.html, letzter Zugriff am 1.12.2020, S. 1.

36) $\operatorname{COM}(2020) 724$ final, S. 6.

37) $\operatorname{COM}(2020) 724$ final, S. 6.

38) $\operatorname{COM}(2020) 724$ final, S. 7.

39) $\operatorname{COM}(2020) 724$ final, S. 7.

40) $\operatorname{COM}(2020) 724$ final, S. 6.

41) $\operatorname{COM}(2020) 724$ final, S. 6.

42) $\operatorname{COM}(2020) 724$ final, S. 6.

43) $\operatorname{COM}(2020) 724$ final, S. 9

44) $\operatorname{COM}(2020) 725$ final, S. 1.

45) Von Eiff, Das Krankenhaus 2020, 870.

46) Im Jahre 2020 wurden 300 Meldungen zu Lieferengpässen gemacht; von Eiff, Das Krankenhaus 2020, 870.

47) $\operatorname{COM}(2020) 724$ final, S. 9.

48) von Eiff, Das Krankenhaus 2020, 870.

49) Art. 168 Abs. 1 S. 1 AEUV.

50) Art. 168 Abs. 1 S. 2 AEUV.

51) Art. 168 Abs. 7 S. 1 AEUV.

52) Art. 168 Abs. 4a AEUV.

53) Art. 168 Abs. 4b AEUV.

54) Art. 168 Abs. 4c AEUV.

55) Kingreen, in: Calliess/Ruffert, EUV/AEUV, 5. Aufl. 2016, AEUV Art. 168 (ex-Art. 152 EGV), Rdnr. 14. 
Versorgung, die stationären Leistungen, das Vergütungssystem, die Qualitätspolitik und die Strukturqualität. Hier ist die Ungleichheit besonders prägnant ${ }^{56}$.

Die nationale Selbstbestimmung und die Notwendigkeit gemeinsamen Handelns stehen der gemeinsamen Zielvereinbarung im Wege. Als Anpassungsmöglichkeit käme eine einheitliche Risikoadjustierung infrage ${ }^{57}$. Sie würde eine vergleichbare Ergebnisqualität medizinischer Interventionen (beispielsweise im Bereich der künstlichen Beatmung von Covid-19-Patienten) gewährleisten.

Gelingt die Harmonisierung auf EU-Ebene nicht, stehen die Mitgliedstaaten den Herausforderungen allein gegenüber. Während der Corona-Krise betraf dies insbesondere die Bereiche der Finanzierung des Gesundheitswesens und die medizinische Versorgung ${ }^{58}$.

Um dem entgegenzuwirken und die Handlungsfähigkeit der einzelnen Mitgliedsstaaten zu stärken, wurde eine Anpassung des EU-Rahmenrechts vorgenommen. Ziel war es, die durch die fehlende Harmonisierung verursachten Stockungen zu beseitigen. Diese Anpassungen beinhalteten, dass die EMA bereits ab der Entwicklung eines möglichen Covid-19 Impfstoffes involviert war und den Entwicklungsprozess engmaschig begleiten und unterstützen konnte ${ }^{59}$. Ein bedingtes Zulassungssystem ermöglichte, dass ein Wirkstoff bei positivem Nutzen-Risiko-Verhältnis zunächst mit weniger umfassenden Daten belegt werden musste, um zugelassen zu werden ${ }^{60}$. Fehlende Daten konnten dann nachträglich noch ergänzt werden. Die so erreichte regulatorische Flexibilität bewirkte eine dringliche Zulassung eines Covid-19-Impfstoffes und beschleunigte den Zulassungsprozess ohne Verlust von Qualitäts- und Sicherheitsstandards ${ }^{61}$.

Die Präsidentin der Europäischen Kommission Ursula von der Leyen forderte in der Rede zur Lage der Union dazu auf, Lehren aus der Krise zu ziehen und diese in einer Europäischen Gesundheitsunion umzusetzen ${ }^{62}$. Unter anderem ist geplant das Überwachungssystem der ECDC zukünftig zu stärken ${ }^{63}$, da die ECDC derzeit nur begrenzte Kapazitäten zur Erfassung einer faktengestützten Momentaufnahme einer Krisenlage hat ${ }^{64}$. Die EMA ist insbesondere wichtig, um Behandlungen und Impfstoffen einzuordnen und zu bewerten ${ }^{65}$. In Zeiten der Krise wurde jedoch deutlich, dass sie nicht in der Lage war, Engpässe bei dringend benötigten Arzneimittellieferungen zu verhindern und die Lieferung und Verteilung ausreichend $\mathrm{zu}$ überwachen ${ }^{66}$. Deshalb muss auch ihre Kompetenz gestärkt werden. AuBerdem soll die Datenübermittlung verbessert werden, damit ein Austausch zwischen den Mitgliedsstaaten über freie Kapazitäten beispielsweise von intensivpflichtigen Patienten oder der Anzahl von freien Krankenhausbetten besteht ${ }^{67}$.

Die benannten Probleme zeigen den Handlungsbedarf in der EU auf und verpflichten zu einer besseren Koordinierung und Bündelung von Ressourcen. Diese Erfahrungen sollen den Grundstock für die geplante Europäische Gesundheitsunion bilden. Inwiefern diese umgesetzt werden sollen und können, wird im Folgenden erläutert.

\section{Ziele der Europäischen Gesundheitsunion - Beschreitung eines neuen Weges?}

Die Europäische Kommission schlägt mehrere Legislativakte zur Schaffung der neuen Europäischen Gesundheitsunion vor ${ }^{68}$. Der rechtliche Rahmen wird durch eine neue Verordnung gebildet. Diese soll die künftige Grundlage bilden, um frühzeitig und umfassend auf grenzüberschreitende Gesundheitsverfahren ${ }^{69}$ reagieren zu können. Die Neuerungen setzen dabei an unterschiedliche Handlungsmechanismen an. Um hierbei eine Übersichtlichkeit zu schaffen, werden sie anhand eines zeitlichen Handlungsstranges erklärt. Auch bei grenzüberschreitenden Ge- sundheitsgefahren gilt „Vorsicht ist besser als Nachsicht“. Darum sind vorverlagerte Handlungen all jene, die Gesundheitsgeschehen beobachten und Vorkehrungen für den Ernstfall treffen, ohne dass bereits eine konkrete Gefahr vorliegt. Anschließend gilt es, Kompetenzen auszuschöpfen und Notfallmechanismen zu aktivieren ${ }^{70}$ sowie auf europäischer Ebene unabhängig von Importen auBerhalb der EU zu sein. Im Nachgang stellen sich Fragen der Verteilung, der Solidarität und der Finanzierung der Krise. Der Forderung nach einer besseren Koordinierung der Politiken der einzelnen Mitgliedstaaten zur Gewährleistung eines hohen Gesundheitsschutzniveaus in ganz Europa $^{71}$ soll die geplante Europäische Gesundheitsunion nun Rechnung tragen.

\section{Vorbereitung auf künftige Gesundheitskrisen}

Vorbereitungen auf eine Krise können vielfältig sein. Kernaufgaben werden aber die Früherkennung von Gesundheitskrisen und ein selbstbestimmtes, unabhängiges Handeln auf europäischer Ebene sein. Die Vorausplanung setzt dort an, wo Handlungsstränge in der Corona-Krise versagt haben.

\section{Ressourcenbildung und Berichterstattung}

Zu Beginn der Krise stand der Schutz des medizinischen Personals im Mittelpunkt, da dieses die Schnittstelle zwischen Gesunden und Kranken bildet. Hierfür war es wichtig, ausreichend persönliche Schutzkleidung zur Verfügung zu stellen. Die Nachfrage nach PSA als Reaktion auf den sofortigen Bedarf konnte weder in Deutschland noch in den anderen Mitgliedstaaten gedeckt werden. Zwar gab es bereits vorher ein Katastrophenschutzsystem, dessen Kapazität war jedoch erreicht, wenn mehrere Mitgliedsstaaten gleichzeitig ein Hilfeersuchen hatten ${ }^{72}$. Für den Ausruf eines Katastrophennotstandes auf ganzer EU-Ebene war das System nicht ausgelegt ${ }^{73}$. Deshalb wurde für den zukünfti-

56) Fischer/Steinhauser/deWever, Das Krankenhaus 2020, 765.

57) Fischer/Steinhauser/deWever, Das Krankenhaus 2020, 765, 766.

58) Bockholdt/Lungstras/Schmidt, NZS 2020, 324f.

59) Mitteilung der Kommission an das Europäische Parlament, den Rat, den Europäischen Wirtschafts- und Sozialausschuss und den Ausschuss der Regionen, EU-Strategie für COVID-19-Impfstoffe, Europäische Kommission, COM(2020) 245 final, S. 7.

60) $\operatorname{COM}(2020) 245$ final, S. 8.

61) $\operatorname{COM}(2020) 245$ final, S. 2

62) Rede von der Leyen vor dem Europäischen Parlament, Building the world we want to live in: A Union of vitality in a world of fragility, https://ec.europa.eu/commission/presscorner/detail/ov/ SPEECH_20_1655, v. 16.9.2020, letzter Zugriff am 8.3.3021. Ausschnitt: „,For me, it is crystal clear - we need to build a stronger European Health Union. And to start making this a reality, we must now draw the first lessons from the health crisis.".

63) $\operatorname{COM}(2020) 724$ final, S. 5.

64) $\operatorname{COM}(2020) 724$ final, S. 5

65) $\operatorname{COM}(2020) 724$ final, S. 5

66) $\operatorname{COM}(2020) 724$ final, S. 5.

67) COM(2020) 724 final, S. 2.; EUCom, Pressemitteilung v. 11.11.2020.

68) EUCom, Pressemitteilung v. 11.11.2020.

69) $\operatorname{COM}(2020) 724$ final, S. 1; EUCom, Pressemitteilung v. 11.11.2020.

70) Deutsche Sozialversicherung Europavertretung, Auf dem Weg zur Gesundheitsunion, v. 1.12.2020, https://dsv-europa.de/ de/news/2020/11/gesundheitsunion.html, letzter Zugriff am 1.12.2020.

71) Vorschlag für eine Verordnung des Europäischen Parlaments und des Rates zu schwerwiegenden grenzüberschreitenden Gesundheitsgefahren und zur Aufhebung des Beschlusses Nr. 1082/2013/EU, Europäische Kommission, COM(2020) 727 final, S. 1.

72) $\operatorname{COM}(2020) 724$ final, S. 10.

73) $\operatorname{COM}(2020) 724$ final, S. 10. 
gen Bedarf ein Vorrat an medizinischer Notfallausrüstung angelegt (rescEU-Reserve) ${ }^{74}$. Die rescEU-Reserve kann nun die Kapazität für den äußersten Notfall im Katastrophenfall der Union decken ${ }^{75}$. Damit soll eine gesteigerte Nachfrage an PSA, Labormaterialien und die Ausstattung für Intensivstationen bedient werden können, die über die übliche nationale Kapazität hinausgeht ${ }^{76}$.

Zur Abschwächung eines Wettbewerbs um notwendige medizinische Güter verstärkt die neue Verordnung den Solidaritätsgrundsatz in der EU. So werden zentrale Vereinbarungen zur gemeinsamen Beschaffung verstärkt und eine „Exklusivitätsklausel“ eingeführt ${ }^{77}$. Diese Exklusivitätsklausel soll Alleingänge verhindern und die Gefahr eines Binnenwettbewerbs minimieren ${ }^{78}$.

Ebenfalls muss die Funktions- und Reaktionsfähigkeit auf Unionsebene getestet werden. Dies gelingt durch die Ausarbeitung, Entwicklung und Umsetzung einer verbindlichen EU-Vorsorge ${ }^{79}$. Der Vorschlag für einen Vorsorge- und Reaktionsplan umfasst die Entwicklung eines Unionsplans für Gesundheitsrisiken zur Förderung einer wirksamen und koordinierten Reaktion ${ }^{80}$. So sollen u.a. eine zeitnahe Zusammenarbeit und ein sicherer Informationsaustausch zwischen der Kommission, den Mitgliedstaaten und den Agenturen der Union ermöglicht werden, wobei der Schwerpunkt bei epidemiologischer Überwachung und Monitoring, frühzeitiger Meldung und Risikobewertung sowie Krisenkommunikation liegt ${ }^{81}$. Zusätzlich müssen die Mitgliedstaaten alle drei Jahre zu nationalen Plänen Bericht erstatten und ggfs. Korrekturmaßnahmen ergreifen $^{82}$. Regelmäßige sektorenübergreifende Stresstests im Gesundheitswesen sind verpflichtend. Dies gilt national wie auch auf EU-Ebene ${ }^{83}$. Aus diesen Erkenntnissen heraus werden Pläne und einschlägige Empfehlungen für Handlungsschritte bei zukünftigen Gesundheitskrisen entwickelt ${ }^{84}$, um eine Harmonisierung von Vorsorge- und Reaktionsplänen zu erreichen ${ }^{85}$. Dadurch können im Krisenfall getroffene ad-hoc-Entscheidungen schneller ineinander greifen.

Das Frühwarn- und Reaktionssystem (engl. Early Warning Response System, EWRS) soll weiter ausgebaut werden. Dieses Schnellwarnsystem nutzt die laufende Kommunikation zwischen Mitgliedstaaten und Kommission, um Gefahren für die öffentliche Sicherheit zu melden und schnell reagieren zu können ${ }^{86}$. In den Mitgliedstaaten sollen ebenfalls mehr Daten zu bevorstehenden möglichen Gesundheitsrisiken erhoben werden. Da Gesundheitskrisen meist grenzüberschreitend wirken und nicht lokal eingrenzbar sind, ist die Einführung einer stetigen epidemiologischen Überwachung und einheitlichen Risikobewertung $^{87}$ geplant. Die obligatorische epidemiologische Überwachung wird dabei Arbeitsbereich der ECDC $\operatorname{sein}^{88}$. Die Überwachung neuartiger Krankheitserreger soll durch den Einsatz künstlicher Intelligenz erfolgen und eine Vergleichbarkeit durch harmonisierte Datensätze und gemeinsame Falldefinition geschaffen werden ${ }^{89}$. Es sollen so neue Risikobewertungsrahmen für alle Gefahren skizziert und zugleich eine Empfehlung der jeweiligen Gegenmaßnahmen mitgegeben werden ${ }^{90}$. Durch einen Ausbau von Testmöglichkeiten (nicht nur auf Covid-19, sondern auch auf andere Gesundheitsgefahren) und eine bessere Kontaktnachverfolgung ist eine bessere strategische Handlung beabsichtigt ${ }^{91}$.

Zukünftig sollen die EMA und die ECDC im Rahmen der begrenzten Kompetenzen ausgebaut werden. Da es bislang keinen Mechanismus gibt, der die Verfügbarkeit von Medizinprodukten in Krisenzeiten überwacht, ist geplant einen solchen neu einzuführen ${ }^{92}$. Die EMA übernimmt künftig u.a. die Überwachung der Arzneimittel und Medizinprodukte, damit Engpässe vermieden wer$\mathrm{den}^{93}$. Sie wird unterstützt durch die Ad-hoc-EMA-Covid-19-Pandemie Task Force, welche bei der Konzeption klinischer Studien und der Produktentwicklung beraten wird $^{94}$. Das ist ein Krisenreaktionsteam, welches ausgesandt werden kann, um bei lokalen Ausbrüchen zu unterstützen ${ }^{95}$. Ziel der Neuerungen soll sein, dass Arzneimittel in Krisenzeiten kurzfristig und ohne langen Vorlauf zugelassen werden können ${ }^{96}$, ohne dass dies zu Lasten der zeitaufwendigen Qualitätssicherung geht. Daran wird auch das ECDC arbeiten, denn es ist geplant diesem einen verbesserten Zugang zu Gesundheitsdaten zu ermöglichen. Die Einführung von IT-Infrastrukturen soll dem besseren Austausch zwischen den Akteuren, insbesondere der EMA und der ECDC, dienen, damit Studien zur klinischen Wirksamkeit besser koordiniert werden können ${ }^{97}$. Wie bekannte Qualitäts- und Sicherheitsstandards einer Krise weichen können, hat man an der Verschiebung der Anwendung der EU-Medizinprodukteverordnung (MDR) erkennen können ${ }^{98}$. Die EU-Kommission wollte dem erhöhten Bedarf an Medizinprodukten nicht mit zusätzlichen Hürden begegnen und so eine ausreichende Verfügbarkeit von wichtigen Krisenmedikamenten sicherstellen ${ }^{99}$. Den Herstellern sollten so Freiräume gegeben werden ${ }^{100}$. Mittlerweile arbeiten die Zulassungs-

74) $\operatorname{COM}(2020) 724$ final, S. 10

75) $\operatorname{COM}(2020) 724$ final, S. 10

76) $\operatorname{COM}(2020) 724$ final, S. 10

77) $\operatorname{COM}(2020) 724$ final, S. 11.

78) So wurde dies schon bei den Ankaufsverfahren der EU angewendet; $\operatorname{COM}(2020) 724$ final, S. 14.

79) $\operatorname{COM}(2020) 724$ final, S. 15

80) Art. 5 Abs. 1 aus dem Vorschlag für eine Verordnung des Europäischen Parlaments und des Rates zu schwerwiegenden grenzüberschreitenden Gesundheitsgefahren und zur Aufhebung des Beschlusses Nr. 1082/2013/EU, Europäische Kommission, COM(2020) 727 final.

81) Art. 5 Abs. 3 aus dem Vorschlag für eine Verordnung, COM(2020) 727 final.

82) $\operatorname{COM}(2020) 724$ final, S. $14 \mathrm{ff}$

83) $\operatorname{COM}(2020) 724$ final, S. 16.

84) Vgl. EUCom, Pressemitteilung v. 11.11.2020.

85) Europäische Kommission, Eine europäische Gesundheitsunion: Gesundheitskrisen gemeinsam bewältigen, 11.11.2020; https:// europainfo.at/download/eine-europaeische-gesundheitsuniongesundheitskrisen-gemeinsam-bewaeltigen-2/, letzter Zugriff am 9.3.2021.

86) $\operatorname{COM}(2020) 724$ final, S. 21.

87) Europäische Kommission, Eine europäische Gesundheitsunion: Gesundheitskrisen gemeinsam bewältigen, 11.11.2020; https:// europainfo.at/download/eine-europaeische-gesundheitsuniongesundheitskrisen-gemeinsam-bewaeltigen-2/, letzter Zugriff am 9.3.2021.

88) $\operatorname{COM}(2020) 724$ final, S. 17

89) $\operatorname{COM}(2020) 724$ final, S. 18

90) COM(2020) 724 final, S. 21.

91) Europäische Kommission, Eine europäische Gesundheitsunion: Gesundheitskrisen gemeinsam bewältigen, 11.11.2020; https:// europainfo.at/download/eine-europaeische-gesundheitsuniongesundheitskrisen-gemeinsam-bewaeltigen-2/, letzter Zugriff am 9.3.2021.

92) $\operatorname{COM(2020)~} 245$ final, S. 2.; COM(2020) 724 final, S. $11 \mathrm{f}$.

93) Vorschlag für eine Verordnung des Europäischen Parlaments und des Rates zu einer verstärkten Rolle der Europäischen Arzneimittel-Agentur bei der Krisenvorsorge und dem Krisenmanagement in Bezug auf Arzneimittel und Medizinprodukte, Europäische Kommission, COM(2020) 725 final, S. 1.

94) $\operatorname{COM}(2020) 725$ final, S. 13; ebenfalls seit dem 1.4.2020.

95) $\operatorname{COM}(2020) 724$ final, S. 23

96) $\operatorname{COM}(2020) 724$ final, S. 12

97) $\operatorname{COM}(2020) 725$ final, S. 15

98) Nachrichten zum Europäischen Sozial- und Arbeitsrecht, ZESAR 2020, 189.

99) Nachrichten zum Europäischen Sozial- und Arbeitsrecht, ZESAR 2020, 189.

100) Nachrichten zum Europäischen Sozial- und Arbeitsrecht, ZESAR 2020, 189. 
stellen mit Sonderzulassungen wie beispielsweise für die am 6.3.2021 durch Discounter deutschlandweit zur Verfügung gestellten Selbsttests ${ }^{101}$.

Im Krisenfall sollen finanzielle Quellen freigesetzt werden, um Fortschritte anzutreiben. Die finanzielle Förderung steht zukünftig aus den EU4Health Programm und Strukturfonds sowie der Forschungs- und Innovationsförderung von Horizont Europa zur Verfügung ${ }^{102}$. Durch die Aktivierung von Soforthilfeinstrumenten soll ein Krisenmodus ausgelöst werden, wodurch schneller auf finanzielle Ressourcen zugegriffen werden kann und Zusagen über noch nicht zugelassene Notfallprodukte erteilt werden können ${ }^{103}$. Dadurch sinken zwar auch die Qualitätssicherungsinstrumente, dies aber zugunsten eines Schnellzulassungsverfahrens.

Um die Entwicklung und Herstellung von krisennotwendigen Produkten zu fördern, wird mit den Entwicklern eine Abnahmegarantie vereinbart. Sie dient als Soforthilfeinstrument, wobei finanzielle Investitionen der Unternehmen belohnt werden ${ }^{104}$. Eine solche Abnahmegarantie wurde bereits für Impfstoffe, welche im Rahmen der Impfstoffstrategie EU entwickelt wurden, abgegeben ${ }^{105}$. Dabei werden zu Beginn der Forschung die groben Eckdaten wie Beitragshöhe, Zeitplan und Finanzierungsstruktur und die Details zur zu Ausgabe, Kosten und Bereitstellung der Impfstoffe festgelegt ${ }^{106}$.

\section{Unabhängigkeit}

Der Wettbewerb und die Lieferengpässe förderten den Wunsch freier von der Medikamentenentwicklung und der Herstellung von PSA im Ausland zu sein. Die Interessenverbände ${ }^{107}$ auf deutscher Seite setzen sich deshalb für mehr Unabhängigkeit bei wichtigen Arzneimitteln und Wirkstoffen von Produktionen in Drittländern ein ${ }^{108}$. Deutschland solle zukünftig weniger auf Importe aus Ländern wie China und Indien angewiesen sein und in einer Krise unabhängiger $\operatorname{sein}^{109}$. Ob dies durch Bevorratung oder eigene Herstellung von lebensnotwendigen Arzneimitteln erfolge, sei nicht entscheidend, wichtig sei eine strategische Unabhängigkeit ${ }^{110}$.

Dies setzte die Europäische Kommission auch im Aufbau der Europäischen Gesundheitsunion um. Eine neue Arzneimittelstrategie soll auf diese Bedürfnisse reagieren ${ }^{111}$, sodass Europa zukünftig selbst über die notwendigen Ressourcen verfügt. Das hat beträchtliche Vorteile. Eine Produktion und Investition in Europa stärkt die Lieferketten und führt zur strategischen Autonomie Europas ${ }^{112}$. Durch die strategische Unabhängigkeit, könnten auch Alleingänge und Exportverbote durch Mitgliedsstaaten und Drittländern wirksam ausgehalten werden ${ }^{113}$. Zugleich ist die Preispolitik weniger vom Wettbewerb und von den Preisen der Zulieferer bestimmt, sodass der Zugang zu innovativen Arzneimitteln zu erschwinglichen Preisen für die EU-Bürger auch im Krisenfall sichergestellt werden $\mathrm{kann}^{114}$. Sollte der Bedarf durch externe Lieferanten abbrechen, so stehen finanzielle Mittel zur Verfügung, um in neue Wirkstoffe in Forschung und Innovation $\mathrm{zu}$ investieren, je nachdem welche gesundheitspolitischen Bedürfnisse auftreten ${ }^{115}$. Die Finanzierung soll gleichzeitig die Digitalisierung wie neue Technologien in der Arzneimittelentwicklung und -herstellung ermöglichen ${ }^{116}$. So dient ein neues Covid-19 Data-Portal ${ }^{117}$ der Sammlung und dem Austausch von Forschungsdaten zu Covid-19 und kann von den Mitgliedstaaten zu Forschungs- und Handlungszwecken genutzt werden. Daneben wurde eine Plattform (Covid-19 Health System Response Monitor, kurz: HSRM) eingerichtet ${ }^{118}$. Sie bildet eine zugängliche Quelle für die Mitgliedstaaten, um sich darüber zu informieren, welche Themen hinsichtlich der Pandemie interessieren und welchen Umgang der Staat damit hat ${ }^{119}$. Da die Plattform auf große Datenquellen verweist, kön- nen die Mitgliedsstaaten so voneinander lernen und ihren eigenen Umgang mit der Krise reflektieren ${ }^{120}$. Es ist also ein Austesten und Offenlegen der Erfahrungen mit der nationalen Handhabung. Das Netzwerk sammelt Daten von Länderexperten, Wissenschaftlern und den jeweiligen WHO-Ländersitzen ${ }^{121}$. Beispielsweise: Wie gelang der Wettbewerb um Personal im Gesundheitswesen, wie können Einnahmeausfälle kompensiert werden oder wie können sich die Gesundheitseinrichtungen vor einem finanziellen Zusammenbruch schützen ${ }^{122}$ ?

\section{Im Ernstfall}

\section{Koordinierung der Handlungsschritte}

Da man einer grenzüberschreitenden Gesundheitsgefahr nur bedingt vorbereitet entgegentreten kann, müssen die Schritte in der Krise so flüssig wie möglich laufen. Das gelingt nur, wenn die Koordinierung der einzelnen Akteure engmaschiger erfolgt, damit einzelne Reaktionen schneller aufeinander abgestimmt werden können ${ }^{123}$. Es ist geplant, dass die EU zukünftig auch ohne enge Abstimmung mit der WHO eine Krisensituation feststellen darf ${ }^{124}$.

Ein großes Thema ist auch die Flexibilität des Handelns im Katastrophenfall. Regulatorische Flexibilität soll zukünftig dazu dienen, dass eine Kontrolle von außen die Versorgung mit notwendigen Arzneimitteln und Medi-

101) Tagesschau, ,Verkaufsstart bei Discountern - Ansturm auf Corona-Selbsttests“, Stand 6.3.2021, https://www.tagesschau.de/ inland/corona-selbsttests-discounter-101.html, letzter Zugriff am 1.7.2021.

102) $\operatorname{COM}(2020) 724$ final, S. 15

103) $\operatorname{COM}(2020) 724$ final, S. 10

104) $\operatorname{COM}(2020) 245$ final, S. 4.

105) $\operatorname{COM}(2020) 724$ final, S. 10.

106) $\operatorname{COM}(2020) 245$ final, S. 4.

107) Deutsche Sozialversicherung Arbeitsgemeinschaft Europa e. V., zusammengesetzt aus der Deutschen Rentenversicherung Bund, Deutsche Gesetzliche Unfallversicherung, der GKVSpitzenverband und die Verbände der gesetzlichen Krankenund Pflegekassen auf Bundesebene.

108) Deutsche Sozialversicherung Europavertretung, Arzneimittel für kommende Generationen - mehr Versorgungssicherheit durch strategische Unabhängigkeit?, Impulspapier, 20. 10.2020, S. 1 (im Folgenden: DSV Europa, Impulspapier, S.).

109) DSV Europa, Impulspapier, S. 1.

110) DSV Europa, Impulspapier, S. 1.

111) DSV Europa, Impulspapier, S. 2.

112) Europäische Kommission, Erschwingliche, zugängliche und sichere Arzneimittel für alle: Die Kommission stellt eine Arzneimittelstrategie für Europa vor, Pressemitteilung, 25.11.2020 (im Folgenden: EUCom, Pressemitteilung v. 25.11.2020).

113) DSV Europa, Impulspapier, S. 2.

114) $\operatorname{COM}(2020) 725$ final, S. 5.

115) EUCom, Pressemitteilung v. 25.11.2020, vgl. Art. 9 Abs. 3 g) Vorschlag für eine Verordnung, COM(2020) 725 final.

116) $\operatorname{COM}(2020) 725$ final, S. 45

117) https://www.covid19dataportal.org/, letzter Zugriff am 11.3. 2021.

118) Merkur/Maresso/Cylus et al., Eurohealth, Vol. 26, No. 2, 2020, S. 6 .

119) Merkur/Maresso/Cylus et al., Eurohealth, Vol. 26, No. 2, 2020, S. 6.

120) Merkur/Maresso/Cylus et al., Eurohealth, Vol. 26, No. 2, 2020, S. 6.

121) Merkur/Maresso/Cylus et al., Eurohealth, Vol. 26, No. 2, 2020, S. 6.

122) Merkur/Maresso/Cylus et al., Eurohealth, Vol. 26, No. 2, 2020 , S. 8 .

123) Deutsche Sozialversicherung Europavertretung, „Auf dem Weg zur Gesundheitsunion“ v. 1.12.2020, https://dsv-europa.de/ de/news/2020/11/gesundheitsunion.html, letzter Zugriff am 1. 12.2020 .

124) $\operatorname{COM}(2020) 724$ final, S. 8. 
zinprodukten überwacht und begleitet, ohne vorherige Sicherheitsstandards zu vernachlässigen ${ }^{125}$. Hierfür werden und wurden Leitlinien ständig aktualisiert, um die Akteure über neue Regularien zu informieren ${ }^{126}$. Durch in Gang setzen des EU-Krisenmechanismus wird der Handlungsspielraum für die EU flexibler. Die Feststellung und Aktivierung des EU-Krisenmechanismus wird von einem weiteren unabhängig beratenden Ausschuss begleitet ${ }^{127}$. Der beratende Ausschuss ist eine Rückversicherungsinstitution, welche eine evidenzbasierte Gesundheitspolitik gewährleisten soll ${ }^{128}$

Die Intensivierung der Zusammenarbeit auf europäischer Ebene und die Schaffung tragfähiger Strukturen soll durch engmaschige Strukturen erreicht werden ${ }^{129}$. Neben der Annäherung von ECDC und EMA sollen auch Informationen zwischen den einzelnen Mitgliedstaaten verstärkt ausgetauscht werden. Anlass war, dass kein verlässlicher und vergleichbarer Überblick auf europäischer Ebene über das Angebot und die Nachfrage an Produkten zur Covid-19 Bekämpfung erstellt werden konnte ${ }^{130}$, wodurch eine nachfragegerechte Umverteilung auf EU-Ebene schwierig wurde. Deshalb verlangt die Ausrufung eines EU-Notstands geradewegs eine engere Koordinierung bei der Aufrüstung mit krisenrelevanten Produkten ${ }^{131}$. Dies erfolgte überwiegend auf private Anfrage hin, wie beispielsweise die Auslastung von grenznahen Kliniken. Zukünftig sollen die Mitgliedsstaaten verpflichtet werden, solche Informationen an die Europäischen Gesundheitsunion zu melden, die anschließend eine Umverteilung veranlassen könnten ${ }^{132}$.

\section{Kompetenzanpassungen}

Das bereits seit $2001^{133}$ bestehende Komitee für Gesundheitssicherheit (Health Security Committee/HSC) ${ }^{134}$ soll ebenfalls Teil des Risikomanagement werden. Der HSC soll an Akzeptanz und Handlungskompetenz zunehmen, denn bislang war die Durchsetzungskraft für gemeinsame Reaktionen der EU und zur Bündelung der Risikokommunikation gering ${ }^{135}$. Auch durch gezielte Empfehlungen für Reaktionsmaßnahmen durch den ECDC sollen diesem mehr Nachdruck verliehen werden, sodass der ECDC koordinierte Reaktionen auf EU-Ebene durchsetzen $\mathrm{kann}^{136}$. Der Gesundheitsausschuss wiederum kann offizielle Leitlinien und Stellungnahmen annehmen ${ }^{137}$. Für den Erfolg der Maßnahme ist entscheidend, dass die Empfehlungen konkrete umsetzbare Maßnahmen enthalten und die Mitgliedsstaaten sich hinsichtlich der Umsetzung verpflichten ${ }^{138}$. Eine EU-Gesundheits-Taskforce kann zur Schulung des Personals im Gesundheitswesens eingesetzt werden $^{139}$.

Neben der Kompetenzerweiterung ist geplant auch neue Kompetenzen auszugestalten. Es soll eine Behörde für die Krisenvorsorge und -reaktion bei gesundheitlichen Notlagen eingerichtet werden ${ }^{140}$. Diese Clearingstelle für medizinisches Gerät der Kommission hilft bei der Abstimmung von Angebot und Nachfrage an Krisengütern und löst Probleme innerhalb der Lieferketten ${ }^{141}$. Auch im Bereich der Entwicklung und Beschaffung biomedizinischer Produkte wird eine neue Behörde eingerichtet, um Versorgungssicherheit bei Arzneimitteln und PSA zu gewährleisten ${ }^{142}$.

Eine weitere EU-Behörde für Krisenvorsorge und -reaktion bei gesundheitlichen Notlagen wird die Health Emergency Preparedness and Response Authority (HERA) $\operatorname{sein}^{143}$. Sie dient der strategischen Früherkennung von Gesundheitsgefahren und soll geeignete Maßnahmen identifizieren ${ }^{144}$. Darunter fällt die Bereitstellung von Infrastruktur für den Datenaustausch und die Bevorratung und den Vertrieb knapper Güter ${ }^{145}$. Die Mitgliedsstaaten sind dadurch in der Lage, im Falle einer gesundheitlichen Notlage unverzüglich medizinische und sonstige Maßnahmen zu ergreifen ${ }^{146}$. Die HERA wird eine weitere Krisenbehörde darstellen, die Synergien und Komplementarität mit den bestehenden EU-Einrichtungen eingehen soll ${ }^{147}$.

\section{Fazit}

Der Vergleich bisheriger Gesundheitspolitik mit den Vorschlägen der Europäischen Gesundheitsunion verdeutlicht die mittlerweile erfolgten Veränderungen. Im Verlauf der Corona-Krise zeigte sich deutlich, dass eine Abstimmung auf europäischer Ebene im Bereich des Gesundheitswesens in der Praxis deutlich stockt. Die bisherige nationale Regelung der Gesundheitssysteme erschwert ein ausgewogenes Miteinander der Mitgliedsstaaten bei grenzüberschreitenden Gesundheitsgefahren. Die Europäische Gesundheitsunion hat viele neue Ansätze, um eine zukünftige Harmonisierung der Abläufe zu erreichen. Die Bereitstellung erheblicher finanzieller Mittel begünstigt die Unabhängigkeit der Europäischen Union im Wettbewerb mit Drittländern und kann im Krisenfall ein weitgehend autonomes Handeln erreichen. Zu bedenken ist allerdings, dass die aus der Corona-Krise gezogenen Lehren vorrangig auf Erfahrungen im Umgang mit dem Covid-19-Virus beruhen. Die Erkenntnisse auf generelle Gesundheitsrisiken zu übertragen, ist nur begrenzt möglich. Wichtig wird sein, sich bei zukünftigen Überlegungen davon frei zu machen und auch andere grenzüberschreitende Gesundheitsrisiken in den Blick zu nehmen. So wird Europa perspektivisch auch lernen müssen Pandemien anderer Art zu bewältigen.

125) $\operatorname{COM}(2020) 724$ final, S. 10

126) $\operatorname{COM}(2020) 724$ final, S. 10

127) $\operatorname{COM}(2020) 724$ final, S. 8.

128) $\operatorname{COM}(2020) 724$ final, S. 8.

129) Europäische Kommission, Eine europäische Gesundheitsunion: Gesundheitskrisen gemeinsam bewältigen, 11.11.2020; https:// europainfo.at/download/eine-europaeische-gesundheitsunion-gesundheitskrisen-gemeinsam-bewaeltigen-2/, letzter $\mathrm{Zu}$ griff am 9.3.2021.

130) $\operatorname{COM}(2020) 724$ final, S. 9.

131) EUCom, Pressemitteilung v. 11.11.2020.

132) EUCom, Pressemitteilung v. 11.11.2020.

133) Damals noch als informelle Beratergruppe zur Gesundheitssicherheit auf europäischer Ebene; https://ec.europa.eu/health/ preparedness_response/risk_management/hsc/members_de.

134) Commission of the European Communities, Commission staff working document, Heath Security in the European Union and Internationally, Brussel, 23.11.2009 SEC(2009) 1622 final.

135) $\operatorname{COM}(2020) 724$ final, S. 8.

136) $\operatorname{COM}(2020) 724$ final, S. 8.

137) $\operatorname{COM}(2020) 724$ final, S. 8.

138) COM(2020) 724 final, S. 8.

139) Europäische Kommission, Eine europäische Gesundheitsunion: Gesundheitskrisen gemeinsam bewältigen, 11.11.2020; https:// europainfo.at/download/eine-europaeische-gesundheitsuniongesundheitskrisen-gemeinsam-bewaeltigen-2/, letzter Zugriff am 9.3.2021.

140) EUCom, Pressemitteilung v. 11.11.2020.

141) $\operatorname{COM}(2020) 724$ final, S. 10.

142) Europäische Kommission, Eine europäische Gesundheitsunion: Gesundheitskrisen gemeinsam bewältigen, 11.11.2020; https:// europainfo.at/download/eine-europaeische-gesundheitsuniongesundheitskrisen-gemeinsam-bewaeltigen-2/, letzter Zugriff am 9.3.2021

143) $\operatorname{COM}(2020) 724$ final, S. 24

144) $\operatorname{COM}(2020) 724$ final, S. 24

145) $\operatorname{COM}(2020) 724$ final, S. 24.

146) $\operatorname{COM}(2020) 724$ final, S. 24.

147) Deutsche Sozialversicherung Europavertretung, ,Auf dem Weg zur Gesundheitsunion“"vom 1.12.2020, https://dsv-europa.de/ de/news/2020/11/gesundheitsunion.html, letzter Zugriff am 1.12.2020, S. 2. 